\title{
Residence of liquids in the infra-junctional portion of the proximal stomach in patients with gastroesophageal reflux disease
}

C.L.A. Barbieri,

L.E.A. Troncon,

J.R.L. Herculano Jr.,

L.R.O. Aprile and

R.O. Dantas
Departamento de Clínica Médica, Faculdade de Medicina de Ribeirão Preto, Universidade de São Paulo, Ribeirão Preto, SP, Brasil

\section{Correspondence \\ L.E.A. Troncon \\ Departamento de Clínica Médica \\ Hospital das Clínicas, FMRP, USP \\ 14048-900 Ribeirão Preto, SP \\ Brasil \\ Fax: +55-16-633-6695 \\ E-mail: ledatron@fmrp.usp.br}

Research supported by CNPq, PRONEX/FINEP, FAPESP, and FAEPA/HCFMRPUSP. C.L.A. Barbieri

was the recipient of an

undergraduate "Introduction to

Science" fellowship from CNPq.

Presented as a "poster of

distinction" at the Digestive

Diseases Week - 2004 (American

Gastroenterological Association)

and published in abstract form in

Gastroenterology 2004; 126

(Suppl 2): A-495 (Abstract T1705).

$\ldots \ldots \ldots \ldots \ldots \ldots$

Received January 27, 2005

Accepted June 21, 2005

\begin{abstract}
Patients with gastroesophageal reflux disease may have disturbances of gastric motility, which could play a role in the pathophysiology of the disease. Recent studies have suggested that the gastric region just below the gastroesophageal junction may have a distinct physiological behavior. We determined whether patients with gastroesophageal reflux disease have abnormal residence of food in the infra-junctional portion of the stomach after ingesting a liquid nutrient meal. Fasted adult patients with reflux disease $(\mathrm{N}=11)$ and healthy volunteers $(\mathrm{N}$ $=10)$ ingested a liquid meal $(320 \mathrm{ml} ; 437 \mathrm{kcal})$ labeled with ${ }^{99 \mathrm{~m}}$ technetium-phytate and their total gastric emptying half-time and regional emptying from the stomach infra-junctional region were determined. In 8 patients, episodes of postprandial acidic reflux to the esophagus were measured for $2 \mathrm{~h}$ using $\mathrm{pH}$ monitoring. There were no differences between reflux patients and controls regarding total gastric emptying time (median: $68 \mathrm{~min}$; range: $39-123 \mathrm{~min} v s 65 \mathrm{~min}$ and 60-99 min, respectively; $\mathrm{P}>0.50$ ). Food residence in the infrajunctional area was similar for patients and controls: 23\% (range: 2030) vs $27 \%$ (range: $19-30 \% ; \mathrm{P}=0.28$ ) and emptying from this area paralleled total gastric emptying $(\mathrm{Rs}=0.79 ; \mathrm{P}=0.04)$. There was no correlation between residence of food in the infra-junctional area and episodes of gastroesophageal reflux $(\mathrm{Rs}=0.06 ; \mathrm{P}=0.88)$. We conclude that it is unlikely that regional motor disturbances involving the infra-junctional region of the stomach play a relevant role in the pathogenesis of acidic gastroesophageal reflux.
\end{abstract}

\section{Introduction}

Gastroesophageal reflux disease (GERD) is a common chronic disease, usually caused by increased episodes of acidic reflux from
Key words

- Gastroesophageal reflux disease

- Gastric emptying

- Gastric motility

- Intragastric distribution

- Proximal stomach 
Studies on animals and man have indicated that the stomach may play a central role in the origin of GERD. For example, gastric distension may elicit transient relaxations of the lower esophageal sphincter (TRLES), which may be associated with increased episodes of gastroesophageal reflux (5-7). Also, delayed gastric emptying, which might cause distension of the stomach, has been reported in substantial proportions of patients with GERD (8-12), although others have failed to confirm this finding (13-16). More recently, it has been shown that patients with GERD may have regional disturbances of gastric motility, such as increased (16-19) or decreased (20) retention of chyme in the proximal stomach, which may occur independently of gastric emptying rates.

Two recent studies exploring the chemical characteristics of the intragastric contents collected from just below the gastroesophageal junction have produced data supporting the view that this area may have a peculiar physiological behavior, distinct from more distal gastric sites (21,22). A study conducted on healthy volunteers reported that, after intragastric administration of nitrates, nitric oxide concentrations over the segment just below the gastroesophageal junction were significantly higher than at all more distal gastric sites (21). In another study from the same group (22) carried out on dyspeptic patients, data from dual gastric and esophageal $\mathrm{pH}$ pull-trough recordings showed a regional variation in intragastric $\mathrm{pH}$, with a "pocket" of more acidic contents just below the gastroesophageal junction (22). It was suggested that the contents of this region escaped the buffering effect of meals, remaining highly acidic in comparison with contents collected from the body of the stomach (22).

We therefore hypothesized that disturbed motor performance of the infra-junctional (IJ) portion of the proximal stomach (the gastric area just below the lower esophageal sphincter) of patients with GERD might lead to accelerated emptying of ingested meals. This would be associated with less buffered, more acidic contents, thus contributing to the pathogenesis of GERD. Hence, characterizing motor abnormalities involving the IJ portion of the proximal stomach might prompt the development of therapeutic strategies focused on this particular anatomical region. The objective of the present study was to determine the postprandial residence of fluids and the emptying rate of the IJ portion of the proximal stomach in GERD patients compared to healthy volunteers.

\section{Material and Methods}

\section{Subjects}

Eleven patients with GERD (4 males and 7 females, median age: 51 years, range: 19 62 years, median body mass index (BMI): $24.6 \mathrm{~kg} / \mathrm{m}^{2}$, range: $19.38-29.82 \mathrm{~kg} / \mathrm{m}^{2}$ ) and 10 healthy, non-symptomatic volunteers from the medical staff and other hospital employees ( 8 males and 2 females) participated in the study after giving written informed consent. Median age in this group was 24.5 years (range: $18-45$ years) and median BMI was $25.22 \mathrm{~kg} / \mathrm{m}^{2}$ (range: 19.71-28.14 kg/ $\mathrm{m}^{2}$ ). There were no significant differences (Student $t$-test) between patients and controls regarding age $(\mathrm{P}>0.10)$ or $\mathrm{BMI}(\mathrm{P}>$ 0.50). The local Ethics Committee approved the study protocol (Statement number 9326/ 00), which was carried out according to the Declaration of Helsinki for research in human beings.

All patients had typical symptoms of GERD (such as heartburn and regurgitation) as predominant complaints. All patients had undergone an upper gastrointestinal tract investigation during a 3-month period preceding the inclusion in the study. This consisted of endoscopy, which was carried out in all patients, and a continuous 24-h ambulatory esophageal $\mathrm{pH}$ recording performed 
in those patients with typical GERD symptoms and a normal endoscopy. On the basis of this investigation, 9 patients were found to have Savary-Miller (23) grade I erosive esophagitis, with no significant $(<2 \mathrm{~cm})$ hiatus hernia, and 2 patients were found to have non-erosive esophagitis. The criteria for inclusion in the GERD group therefore consisted of at least one cardinal GERD symptom (3) plus either erosive esophagitis at endoscopy (23) or abnormal results of the esophageal $\mathrm{pH}$ study $(3,4)$. Criteria for inclusion in the control group consisted of the absence of any active symptom or history of digestive complaints or diseases. None of the subjects included in the study were smokers, had evidence of other diseases or had previous abdominal operations. None of the healthy controls used any drugs. For the GERD patient group, all medications in use were discontinued at least one week before the tests.

\section{Study protocol}

Gastric emptying and the intragastric distribution of a radio-labeled liquid nutrient meal were assessed in all patients and control volunteers by external abdominal scintigraphy. In 8 of the GERD patients, a 2-h postprandial ambulatory esophageal $\mathrm{pH}$ recording using the same test meal was performed in another session carried out within 2-7 days after the first study. All tests were carried out in the morning, after an overnight fast.

Determination of gastric emptying and meal residence in the proximal stomach and in the infra-junctional region

Subjects ingested $320 \mathrm{ml}$ of a liquid test meal containing $437 \mathrm{kcal}$ (64 g carbohydrate, $20 \mathrm{~g}$ protein, and $11 \mathrm{~g}$ fat, $800 \mathrm{mOsm} /$ $\mathrm{kg}$ ) and prepared as described elsewhere (20). Before ingestion, the test meal was labeled with $18 \mathrm{MBq}{ }^{99}$ technetium coupled with phytate (Phytosyd; Sydma Medical Equipments and Reagents, Ribeirão Preto, SP, Brazil) as a non-absorbable carrier. Immediately after meal ingestion, images of the radioactivity in the anterior and posterior aspects of the abdomen were simultaneously collected (30 s of acquisition time) from subjects standing between the low energy, high sensitivity collimators of a dual-headed gamma camera (Sopha Vision, model DST, "Sophycamera", Sopha Medical Vision America, Twinsburg, OH, USA). The camera was set up to record activity with a $20 \%$ window around the $140-\mathrm{keV}$ photo peak of technetium. The acquisition apparatus was connected to an image processing system. Further serial images of the abdomen were then taken every 5 min during the first 20 min, and then at 10-min intervals up to 90 min. In the intervals between image acquisitions subjects sat comfortably in a chair near the camera.

Images corresponding to the anterior projection of the stomach were identified from the frames collected after meal ingestion. A region of interest (ROI) corresponding to the total stomach was outlined with a cursor over the largest anterior gastric image obtained from the first three acquisitions (at 0 , 5 , or 10 min after meal), in which gastric accommodation was expected to be maximal. This ROI was then subdivided into two regions respectively corresponding to the proximal and distal gastric areas, as described elsewhere (20). Additionally, a specific ROI for the IJ area was defined by dividing the ROI for the proximal stomach into two halves. The ROIs corresponding to each region (total, proximal and distal stomach, and IJ) were copied and inverted, so they could fit the posterior images. Counts obtained throughout the study from all anterior and posterior ROIs were recorded and stored. These data were then corrected for physical isotope decay, and the geometric mean of the anterior and posterior counts was computed to correct for the effect of the poste- 
rior-to-anterior movement of the marker within the body.

For each region, "activity versus time" curves, expressed as percentages of activity in the total stomach immediately after the end of meal ingestion, were obtained. These curves permit the calculation of the following variables: gastric emptying half-time $\left(\mathrm{T}_{1 /}\right.$ ${ }_{2}$ ), the time ( $\mathrm{min}$ ) needed for the initial activity in the total stomach to fall by $50 \%$; meal residence in the proximal stomach throughout gastric emptying: the ratio between the average retention of radioactivity in the ROIs corresponding to the proximal and the total stomach. Meal residence in the IJ area throughout gastric emptying was defined as the ratio between the average retention of radioactivity in the ROI corresponding to the IJ area and in both the proximal and total stomach. IJ area emptying half-time $\left(\mathrm{T}_{1 / 2}\right)$ was defined as the time ( $\mathrm{min}$ ) needed for the initial activity in this area to fall by $50 \%$.

\section{Determination of gastroesophageal reflux}

Patients underwent conventional manometry (24) and immediately after the procedure a $\mathrm{pH}$ probe (Synetics Multi-use $\mathrm{pH}$ Catheter; Synetics Medical, Stockholm, Sweden) with a single antimony electrode was introduced so that its tip was positioned $5 \mathrm{~cm}$ above the lower esophageal sphincter. The probe was connected to a $\mathrm{pH}$ meter (Digitrapper MkIII, Synetics Medical) and a basal recording was obtained for $45 \mathrm{~min}$. The patient was then fed the same liquid test meal as used in the abdominal scintigraphy,

Figure 1. Gastric emptying halftimes (total $T_{1 / 2}$ ) for patients with gastroesophageal reflux disease (GERD) and for control subjects after a liquid nutrient meal. The horizontal bars indicate the medians for patients $(\mathrm{N}$ $=11)$ and controls $(\mathrm{N}=10) . \mathrm{P}>$ 0.05 (Mann-Whitney test).

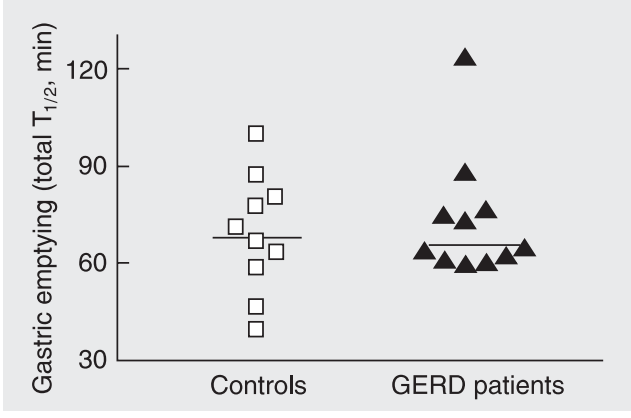

but without adding the radio marker. After ingestion, the patient sat comfortably and was instructed to stand up for one min every 10 min up to $2 \mathrm{~h}$, in order to simulate the variations of body position observed during the isotope study.

The number of acidic reflux episodes during this study was counted after visual inspection of each individual recording using a dedicated software (Polygram function testing for Windows, Synetics Medical). An acidic reflux episode was defined as a fall in esophageal $\mathrm{pH}$ to below the arbitrary limit of $4 \mathrm{pH}$ units for at least $10 \mathrm{~s}$. A further drop of at least $1 \mathrm{pH}$ unit when esophageal $\mathrm{pH}$ was already below 4.0 was considered to be a new-reflux episode (20).

\section{Statistical analysis}

Data are reported as median and range and were analyzed by nonparametric tests since the data did not seem to have a normal distribution. Differences between controls and patients were analyzed by the MannWhitney test. Except for the analysis regarding gastric emptying, the two-tailed test was utilized. The Spearman rank correlation coefficient was employed for the assessment of the relationships between the various gastric motor variables, as well as between residence of liquid in the IJ area and the number of postprandial acidic reflux episodes. Differences were considered to be statistically significant when $\mathrm{P}<0.05$.

\section{Results}

As shown in Figure 1, there was no significant difference between GERD patients and controls regarding total gastric emptying $\mathrm{T}_{1 / 2}$ values (median: $68 \mathrm{~min}$; range: 39123 vs $65 \mathrm{~min}$ and 60-99 min, respectively).

Data regarding residence of food in the proximal stomach are presented in Figure 2, which also shows that there was no significant difference between GERD patients and 
the controls concerning this variable (46\%; 38-60 vs 52\%; 39-59\%; $\mathrm{P}>0.50)$.

Residence of food in the IJ area throughout gastric emptying in GERD patients was similar to that observed in controls, both when expressed as a fraction of total $(23 \%$; $20-30$ vs $26.5 \%$; $19-30 \%$; $\mathrm{P}>0.20)$ or proximal stomach residence $(50.6 \% ; 46.1-54.4 v s$ $50.5 \% ; 48.0-53.1 \%$; $\mathrm{P}>0.50)$. These data are presented in Figure $3 \mathrm{~A}$ and B. Values for the emptying $\mathrm{T}_{1 / 2}$ of the IJ area of GERD patients, although slightly lower, were also not significantly different from those obtained for the control group (58 min; 37-90 vs $64.5 \mathrm{~min}$; 38-85 min; $\mathrm{P}>0.50$ ), as shown in Figure 4. Data in Table 1 shows that, in both groups, the motor behavior of the IJ area paralleled those of both the total and the proximal stomach since highly significant values for the correlation coefficient between the relevant variables were observed. The relationship between the rates of emptying of the IJ area and the total stomach is shown in Figure 5.

In GERD patients, there was no significant correlation between residence of food in the IJ area and the number of acidic gastroesophageal reflux episodes $(\mathrm{Rs}=0.06 ; \mathrm{P}$ $=0.88$ ), as shown in Figure 6 .

\section{Discussion}

Our findings show that, after a liquid nutrient meal, the residence of chyme in the gastric region just below the lower esophageal sphincter (IJ area) parallels the residence rates of both the proximal and total stomach. Indeed, the median values for meal residence in the IJ area of both groups were numerically close to those corresponding to the size of the ROI for that area, when expressed in proportion of either the proximal $(\sim 50 \%)$ or the total stomach $(\sim 25 \%)$. Also, no differences were found between GERD patients and healthy volunteers. Taking together, these findings indicated that it is unlikely that an abnormal motor behavior of the IJ region is involved in the pathogenesis of acidic gastroesophageal reflux.

Based on studies showing that patients with GERD may have regional gastric motor abnormalities (16-20) and that the IJ area of
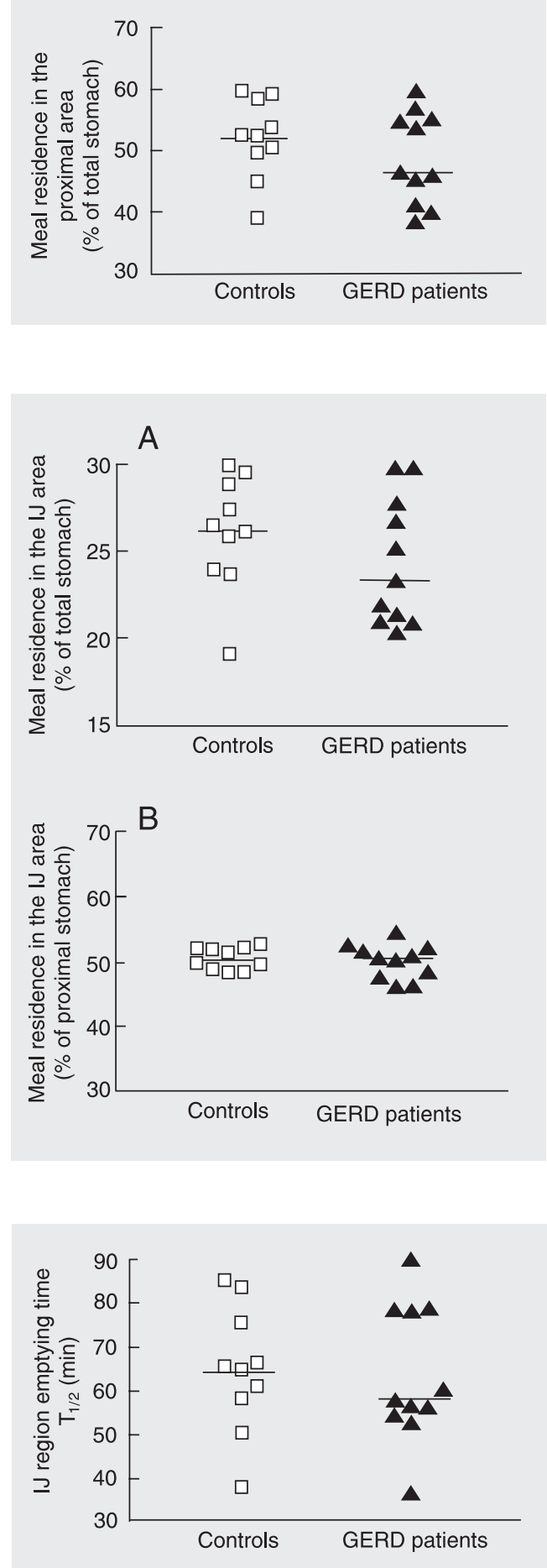

Figure 2. Residence of food in the proximal stomach (reported as a fraction of that for the total stomach) throughout gastric emptying in patients with gastroesophageal reflux disease (GERD) and control subjects, after a liquid nutrient meal. The horizontal bars indicate the medians for patients $(\mathrm{N}=11)$ and controls $(N=10) . P>0.05$ (Mann-Whitney test).

Figure 3. Residence of food in the infra-junctional (IJ) region of the proximal stomach (the area just below the lower esophageal sphincter) throughout gastric emptying in patients with gastroesophageal reflux disease (GERD) and control subjects, after a liquid nutrient meal. Data are reported as fractions of both total (A) and proximal stomach (B) residence values. The horizontal bars indicate the medians for patients $(N=11)$ and controls $(\mathrm{N}=10)$. A, $\mathrm{P}>0.05$; $\mathrm{B}, \mathrm{P}>$ 0.05 (Mann-Whitney test).

Figure 4. Emptying half-time $\left(T_{1 / 2}\right)$ of the infra-junctional (IJ) region of the proximal stomach (the area just below the lower esophageal sphincter) in patients with gastroesophageal reflux disease (GERD) and control subjects, after a liquid nutrient meal. The horizontal bars indicate the medians for patients $(\mathrm{N}=11)$ and controls $(\mathrm{N}=10)$. $\mathrm{P}$ $>0.05$ (Mann-Whitney test). 
the proximal stomach might show a peculiar physiological behavior $(21,22)$, we hypothesized that meal emptying from this area might be accelerated, leading to less buffered and more acidic contents (22). Since transfer of liquids from the proximal to the distal stomach appears to be dependent on gastric fundal pressure (25), abnormally rapid chyme emptying from the IJ area may be associated with increased pressure. This in turn may stretch the cardiac region of the

Table 1. Application of Spearman's correlation coefficient to motor variables related to the infra-junctional (IJ) region of the stomach and those related to both the proximal and the total stomach in patients with gastroesophageal reflux disease (GERD) and in healthy volunteers (controls).

\begin{tabular}{lll}
\hline Subjects & $\begin{array}{c}\text { Emptying } \mathrm{T}_{1 / 2}-\mathrm{IJ} \text { vs } \\
\text { total stomach }\end{array}$ & $\begin{array}{l}\text { Residence }- \text { IJ vs } \\
\text { proximal stomach }\end{array}$ \\
\hline GERD $(\mathrm{N}=11)$ & $\mathrm{Rs}=0.71(0.018)$ & $\mathrm{Rs}=0.95(<0.001)$ \\
Controls $(\mathrm{N}=10)$ & $\mathrm{Rs}=0.88(0.001)$ & $\mathrm{Rs}=0.94(0.0002)$
\end{tabular}

Data in parentheses correspond to the relevant $\mathrm{P}$ values, for the hypothesis that the correlation is significantly different from zero. $T_{1 / 2}$ is the emptying half-time for each region.

Figure 5. Correlation between emptying rates from the infrajunctional (IJ) region of the proximal stomach (the area just below the lower esophageal sphincter) and from the total stomach in patients with gastroesophageal reflux disease, after a liquid nutrient meal. Data are reported for $\mathrm{N}=11$ patients. Rs $=0.71$ and $\mathrm{P}=0.018$ (Spearman's correlation).

Figure 6. Correlation between values for residence of food in the infra-junctional (IJ) region of the proximal stomach (the area just below the lower esophageal sphincter) and the number of acidic gastroesophageal reflux episodes in patients with gastroesophageal reflux disease, after a liquid nutrient meal. $\mathrm{Rs}=0.06$ and $\mathrm{P}>0.05$ (Spearman's correlation).
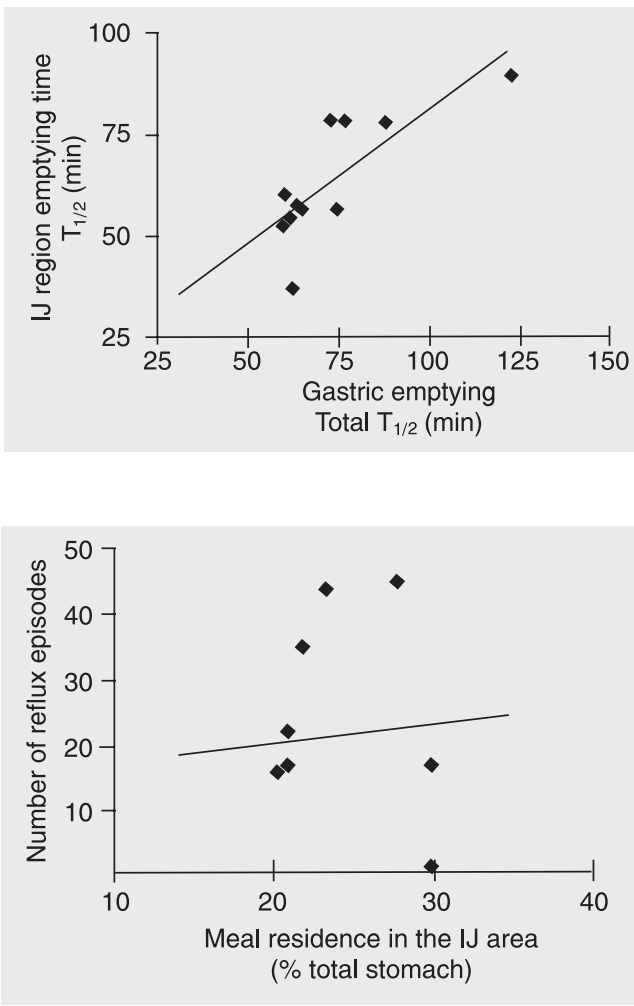

proximal stomach and may be associated with increased rates of both TRLES (1) and reflux episodes $(4,5)$. Nevertheless, we were unable to find any evidence of rapid emptying from this region, or any significant correlation between residence of food in the IJ area and the number of acidic gastroesophageal reflux episodes.

Our patients probably are a rather representative sample of GERD, since this group included only patients with mild or no esophagitis and without hiatus hernia, but with pathological reflux, as demonstrated by $24-$ $\mathrm{h}$ esophageal $\mathrm{pH}$ monitoring. This kind of patient corresponds to the largest contingent in the clinical spectrum of GERD $(1,4)$, and also represents a group in which TRLES originating in the stomach is regarded as a major pathogenic mechanism of gastroesophageal reflux $(1,4,26,27)$.

As far as the test meal employed in our study is concerned, we regard it as suitable for testing the proposed hypothesis of diminished residence of liquids in the IJ region, given its relatively high calorie content and osmolarity, and its ability to elicit a substantial number of postprandial episodes of acidic gastroesophageal reflux, as seen in a previous study with the same meal (20). Nevertheless, we cannot rule out the possibility that using a composite, mixed solidliquid test meal, or more exclusively, a usual solid meal might produce different results.

Measurements of total and regional gastric emptying and the number of acidic gastroesophageal reflux episodes were performed on two different days. However, this is unlikely to be relevant for our negative findings, since the studies were performed under similar conditions, the interval between the two studies was rather short and both scintigraphy (28) and esophageal $\mathrm{pH}$ monitoring (29) are quite reproducible techniques.

Another potential methodological limitation of the present study is the definition of a possibly too large ROI for the IJ region of 
the proximal stomach. This was done so that the longitudinal diameter of this ROI would correspond to $50 \%$ of that for the total stomach, in order to reduce the chance of missing the emergence of the esophagus into the stomach, which may vary from one individual to another. However, this relatively large size may have interfered with the demonstration of more subtle regional motor abnormalities regarding the IJ area. Nevertheless, our findings of preserved emptying from the IJ area agree with those reported in a very recent study which used a different approach to define the IJ area (30). In this study, Sifrim et al. (30) used a radioactive marker to precisely define the gastroesophageal junction and a ROI as small as $10 \%$ of the total stomach around the marker corresponding to this particular gastric region and did not find any abnormal retention in this area.

The lack of a difference between GERD patients and controls for gastric emptying time in the present study differs from other studies showing delayed gastric emptying (8-12). Nevertheless, our findings agree with previous studies that either failed to demonstrate an abnormality in GERD, or found that only a small proportion of patients had delayed emptying $(13-16,20)$. Indeed, close inspection of our data (Figure 1) shows that only one of the 11 GERD patients had an exceedingly high gastric emptying $\mathrm{T}_{1 / 2}$. Also, we did not find differences between GERD patients and controls regarding meal residence in the proximal stomach, which is in contrast to others who reported either in- creased postprandial proximal residence of food (16-19) or diminished retention in the proximal stomach of the same liquid meal as used here (20). However, it is possible to assume that differences between our findings and other studies that demonstrated delayed gastric emptying or postprandial intragastric food maldistribution in GERD are related to different criteria for patient inclusion or diversity in patient demographic and clinical characteristics. Also, it is difficult to rule out that the relatively small number of patients included in the present study may have contributed to the absence of difference between the GERD and control groups, as well as to the discrepancy between our findings and those from other studies (1320).

The present study did not demonstrate any differences between GERD patients and healthy volunteers regarding meal residence in the infra-junctional portion of the proximal stomach. Our results therefore do not support the hypothesis that physiological motor abnormalities in the gastric area located just below the lower esophageal sphincter play a relevant role in the pathogenesis of acidic gastroesophageal reflux.

\section{Acknowledgments}

The authors wish to thank Miss Marie Secaf for excellent technical assistance and Professor Daniel Sifrim, Leuven Catholic University, Leuven, Belgium, for his intellectual contribution to this study.

\section{References}

1. Galmiche JP \& Janssens J (1995). The pathophysiology of gastrooesophageal reflux disease: an overview. Scandinavian Journal of Gastroenterology, 30 (Suppl 211): 7-18.

2. Koelz HR, Blum AL \& The Swiss Esophagitis Study Group (1986). Cardinal symptoms of reflux esophagitis. Gastroenterology, 90 (Suppl 2): 1498 (Abstract).

3. Stacher G \& Weiss W (1989). Diagnosis of gastro-esophageal reflux disease. Scandinavian Journal of Gastroenterology, 24 (Suppl 156):
21-24.

4. Richer JE \& Castell DO (1982). Gastro-esophageal reflux. Annals of Internal Medicine, 97: 93-103.

5. Holloway RH, Hongo M, Berger K et al. (1985). Gastric distention: A mechanism for post-prandial gastroesophageal reflux. Gastroenterology, 89: 779-784.

6. Franzi SJ, Martin CJ, Cox MR et al. (1990). Response of canine lower esophageal sphincter to gastric distention. American Journal 
of Physiology, 259 (Part 1): G380-G385.

7. Boulant J, Fioramonti J, Dapoigny M et al. (1994). Cholecystokinin and nitric oxide in transient lower esophageal sphincter relaxation to gastric distention in dogs. Gastroenterology, 107: 1059-1066.

8. McCallum RW, Berkowitz DM \& Lerner E (1981). Gastric emptying in patients with gastro-oesophageal reflux. Gastroenterology, 80: 285-291.

9. Maddern GJ, Chatterton BE, Collins PJ et al. (1985). Solid and liquid gastric emptying in patients with gastro-oesophageal reflux. British Journal of Surgery, 72: 344-347.

10. Cunningham KM, Horowitz M, Riddell PS et al. (1991). Relations among autonomic nerve dysfunction, oesophageal motility, and gastric emptying in gastro-oesophageal reflux disease. Gut, 32: 14361440.

11. Scarpignato C \& Farneze A (1992). Esophageal exposure to acid in GERD patients with and without delayed gastric emptying. Effect of cisapride. Hepatogastroenterology, 39: 91-92.

12. Benini L, Sembenini C, Castellani G et al. (1996). Gastric emptying and dyspeptic symptoms in patients with gastro-esophageal reflux. American Journal of Gastroenterology, 91: 1351-1354.

13. Csendes A \& Henriquez A (1978). Gastric emptying in patients with reflux oesophagitis or benign strictures of the esophagus secondary to reflux compared to controls. Scandinavian Journal of Gastroenterology, 13: 205-207.

14. Shay SS, Eggli D, McDonald C et al. (1987). Gastric emptying of solid food in patients with symptomatic gastroesophageal reflux. Gastroenterology, 92: 459-465.

15. Keshavarzian A, Bushnell DL, Sontag S et al. (1991). Gastric emptying in patients with severe reflux esophagitis. American Journal of Gastroenterology, 86: 738-742.

16. Stacher G, Lenglinger J, Bergmann $\mathrm{H}$ et al. (2000). Gastric emptying: a contributory factor in gastro-oesophageal reflux activity? Gut, 47: 661-666.

17. Penagini R, Hebbard G, Horowitz M et al. (1998). Motor function of proximal stomach and visceral perception in gastro-oesophageal reflux disease. Gut, 42: 251-257.

18. Tefera S, Gilja OH, Hatlebakk JG et al. (2001). Gastric accommodation studied by ultrasonography in patients with reflux esophagitis. Digestive Diseases and Sciences, 46: 618-625.

19. Tefera S, Gilja OH, Olafsdottir E et al. (2002). Intragastric maldistri- bution of a liquid meal in patients with reflux oesophagitis assessed by three-dimensional ultrasonography. Gut, 50: 153-158.

20. Herculano Jr JRL, Troncon LEA, Aprile LRO et al. (2004). Diminished retention of food in the proximal stomach correlates with increased acidic reflux in patients with gastroesophageal reflux disease and dyspeptic symptoms. Digestive Diseases and Sciences, 49: 750-756.

21. lijima K, Henry E, Moriya A et al. (2002). Dietary nitrate generates potentially mutagenic concentration of nitric oxide at the gastrooesophageal junction. Gastroenterology, 122: 1248-1257.

22. Fletcher J, Wirz A, Young J et al. (2001). Unbuffered highly acidic gastric juice exists at the gastro-esophageal junction after a meal. Gastroenterology, 121: 775-783.

23. Savary M \& Miller G (1978). The esophagus. In: Savary M \& Miller G (Editors), Handbook and Atlas of Endoscopy. Gassman Verlag AG, Solothurn, Switzerland.

24. Dantas RO \& Mamede RCM (1996). Esophageal motility in patients with esophageal caustic injury. American Journal of Gastroenterology, 91: 1157-1161.

25. Mayer EA (1994). The physiology of gastric storage and emptying. In: Johnson LR (Editor), Handbook of Physiology. Raven Press, New York, 929-976.

26. Mittal RK \& McCallum RW (1988). Characteristics and frequency of transient relaxations of the lower esophageal sphincter in patients with reflux esophagitis. Gastroenterology, 95: 593-599.

27. Holloway $\mathrm{RH}$, Kocyan $\mathrm{P}$ \& Dent $\mathrm{J}$ (1991). Provocation of transient lower esophageal sphincter relaxations by meals in patients with symptomatic gastroesophageal reflux. Digestive Diseases and Sciences, 36: 1434-1439.

28. Tosetti C, Paternicó A, Stanghellini V et al. (1998). Reproducibility of a solid and of a liquid caloric meal for gastric emptying studies. Nuclear Medicine Communications, 19: 581-586.

29. Streets CG \& DeMeester TR (2003). Ambulatory 24-hour esophageal pH monitoring: why, when, and what to do. Journal of Clinical Gastroenterology, 37: 14-22.

30. Sifrim D, Verfaille M, Flamen P et al. (2004). Simultaneous assessment of gastric emptying, postprandial gastric acid secretion and acid or weakly acidic gastro esophageal reflux. Gastroenterology, 126 (Suppl 2): A-497 (Abstract T1715). 